Swarthmore College

Works

4-1-2009

\title{
Spectroscopic Measurements Of Temperature And Plasma Impurity Concentration During Magnetic Reconnection At The Swarthmore Spheromak Experiment
}

Vernon Hampden Chaplin , '07

Michael R. Brown

Swarthmore College, doc@swarthmore.edu

David H. Cohen

Swarthmore College, dcohen1@swarthmore.edu

T. Gray

C. D. Cothran

Follow this and additional works at: https://works.swarthmore.edu/fac-physics

Part of the Astrophysics and Astronomy Commons, and the Physics Commons

Let us know how access to these works benefits you

\section{Recommended Citation}

Vernon Hampden Chaplin , '07; Michael R. Brown; David H. Cohen; T. Gray; and C. D. Cothran. (2009). "Spectroscopic Measurements Of Temperature And Plasma Impurity Concentration During Magnetic Reconnection At The Swarthmore Spheromak Experiment". Physics Of Plasmas. Volume 16, Issue 4. DOI: 10.1063/1.3099603

https://works.swarthmore.edu/fac-physics/21

This work is brought to you for free by Swarthmore College Libraries' Works. It has been accepted for inclusion in Physics \& Astronomy Faculty Works by an authorized administrator of Works. For more information, please contact myworks@swarthmore.edu. 


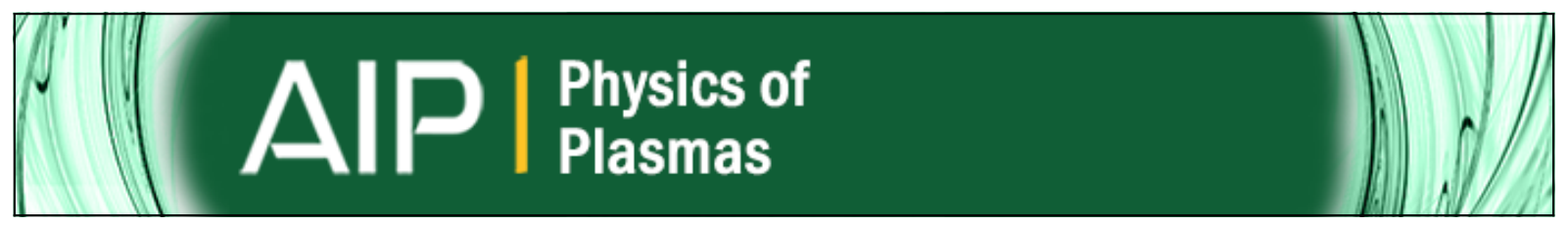

Spectroscopic measurements of temperature and plasma impurity concentration during magnetic reconnection at the Swarthmore Spheromak Experiment

V. H. Chaplin, M. R. Brown, D. H. Cohen, T. Gray, and C. D. Cothran

Citation: Physics of Plasmas (1994-present) 16, 042505 (2009); doi: 10.1063/1.3099603

View online: http://dx.doi.org/10.1063/1.3099603

View Table of Contents: http://scitation.aip.org/content/aip/journal/pop/16/4?ver=pdfcov

Published by the AIP Publishing

\section{AAlP $P$ Re-register for Table of Content Alerts}




\title{
Spectroscopic measurements of temperature and plasma impurity concentration during magnetic reconnection at the Swarthmore Spheromak Experiment
}

\author{
V. H. Chaplin, ${ }^{1,2}$ M. R. Brown, ${ }^{1}$ D. H. Cohen, ${ }^{1}$ T. Gray, ${ }^{1}$ and C. D. Cothran ${ }^{1, a)}$ \\ ${ }^{1}$ Department of Physics and Astronomy, Swarthmore College, Swarthmore, Pennsylvania 19081, USA \\ ${ }^{2}$ California Institute of Technology, Pasadena, California 91125, USA
}

(Received 4 November 2008; accepted 25 February 2009; published online 7 April 2009)

\begin{abstract}
Electron temperature measurements during counterhelicity spheromak merging studies at the Swarthmore Spheromak Experiment (SSX) [M. R. Brown, Phys. Plasmas 6, 1717 (1999)] are presented. VUV monochromator measurements of impurity emission lines are compared with model spectra produced by the non-LTE excitation kinematics code PRISMSPECT [J. J. MacFarlane et al., in Proceedings of the Third Conference on Inertial Fusion Science and Applications (2004)] to yield the electron temperature in the plasma with $1 \mu \mathrm{s}$ time resolution. Average $T_{e}$ is seen to increase from 12 to $19 \mathrm{eV}$ during spheromak merging. Average C III ion temperature, measured with a new ion Doppler spectrometer (IDS) [C. D. Cothran et al., Rev. Sci. Instrum. 77, 063504 (2006)], likewise rises during spheromak merging, peaking at $\sim 22 \mathrm{eV}$, but a similar increase in $T_{i}$ is seen during single spheromak discharges with no merging. The VUV emission line measurements are also used to constrain the concentrations of various impurities in the SSX plasma, which are dominated by carbon, but include some oxygen and nitrogen. A burst of soft x-ray emission is seen during reconnection with a new four-channel detector (SXR). There is evidence for spectral changes in the soft x-ray emission as reconnection progresses, although our single-temperature equilibrium spectral models are not able to provide adequate fits to all the SXR data. (c) 2009 American Institute of Physics. [DOI: 10.1063/1.3099603]
\end{abstract}

\section{INTRODUCTION}

Magnetic reconnection is the process driving the dynamics in spheromak merging and relaxation ${ }^{1}$ as well as in several astrophysical scenarios. ${ }^{2}$ During reconnection, magnetic energy is rapidly converted to electron and ion heat, plasma flow, and energetic particle beams. ${ }^{3,4}$ However, the partitioning of energy among various channels is not fully understood. Spheromak dynamics have been studied at the Swarthmore Spheromak Experiment (SSX) in a number of geometries. ${ }^{2,5-8}$ We have recently been studying spheromak merging in a prolate $0.4 \mathrm{~m}$ diameter, $0.6 \mathrm{~m}$ length, $3 \mathrm{~mm}$ wall copper flux conserver at SSX (see Fig. 1). In these experiments, merging of a pair of counterhelicity spheromaks generates magnetic reconnection dynamics near the midplane. We utilize measurements of UV and x-ray impurity emission spectra to monitor the plasma composition and electron and ion temperatures during and after reconnection.

Spectroscopy has recently been employed to measure $T_{e}$, $T_{i}$, and other plasma properties in a wide range of contexts, from tokamak and reversed field pinch experiments ${ }^{9-11}$ to high-temperature gold plasmas. ${ }^{12}$ In magnetically confined fusion plasmas, impurity species are monitored by measuring their UV and x-ray line emission. ${ }^{13-15}$ Spectroscopic techniques have been also been employed on warm $(T<100$ $\mathrm{eV})$ laboratory plasmas with properties similar to the SSX plasma to measure temperatures and ion flow. ${ }^{16,17}$ In many

\footnotetext{
${ }^{a)}$ Present address: Global Strategies Group, North America, 2200 Defense Highway, Crofton, MD 21114, USA.
}

applications, computer models of radiative processes can be a valuable aid in the interpretation of the spectroscopic data. ${ }^{18,19}$

There are similar applications in astrophysics, especially since the launch of Chandra ${ }^{20}$ and XMM-Newton, ${ }^{21}$ which made high-resolution x-ray spectroscopy of astrophysical plasmas possible. Soft $\mathrm{x}$-ray emission line ratio diagnostics measured with the spectrometers ${ }^{22-24}$ on these satellite observatories are routinely used as probes of the electron temperature in stellar coronae $\mathrm{e}^{25}$ and the winds of massive stars ${ }^{26}$ among other highly ionized astrophysical plasmas. Global spectral modeling, in which a detailed model spectrum, including numerous emission lines and recombination and bremsstrahlung continua, is fit to broadband spectral data, is also being applied to the analysis of solar UV spectra ${ }^{27}$ and stellar x-ray spectra. ${ }^{28-30}$ Both the individual line intensity modeling and the global spectral modeling are also used to determine elemental abundances in astrophysical plasmas. ${ }^{28,29}$ The work we report on here, in the laboratory context, similarly employs both line ratio diagnostics and global spectral modeling of UV and soft x-ray spectra in order to determine electron temperatures and plasma impurity concentrations ("elemental abundances" in the astrophysical parlance). The Chandra and XMM-Newton spectrographs have soft x-ray responses only above about $0.5 \mathrm{keV}$, and so are generally used to probe plasma temperatures a factor of several hotter than those we discuss in this paper. However, the densities, impurity concentrations, dominant atomic processes, and diagnostic techniques are similar.

Local and global magnetic structure of SSX spheromaks 


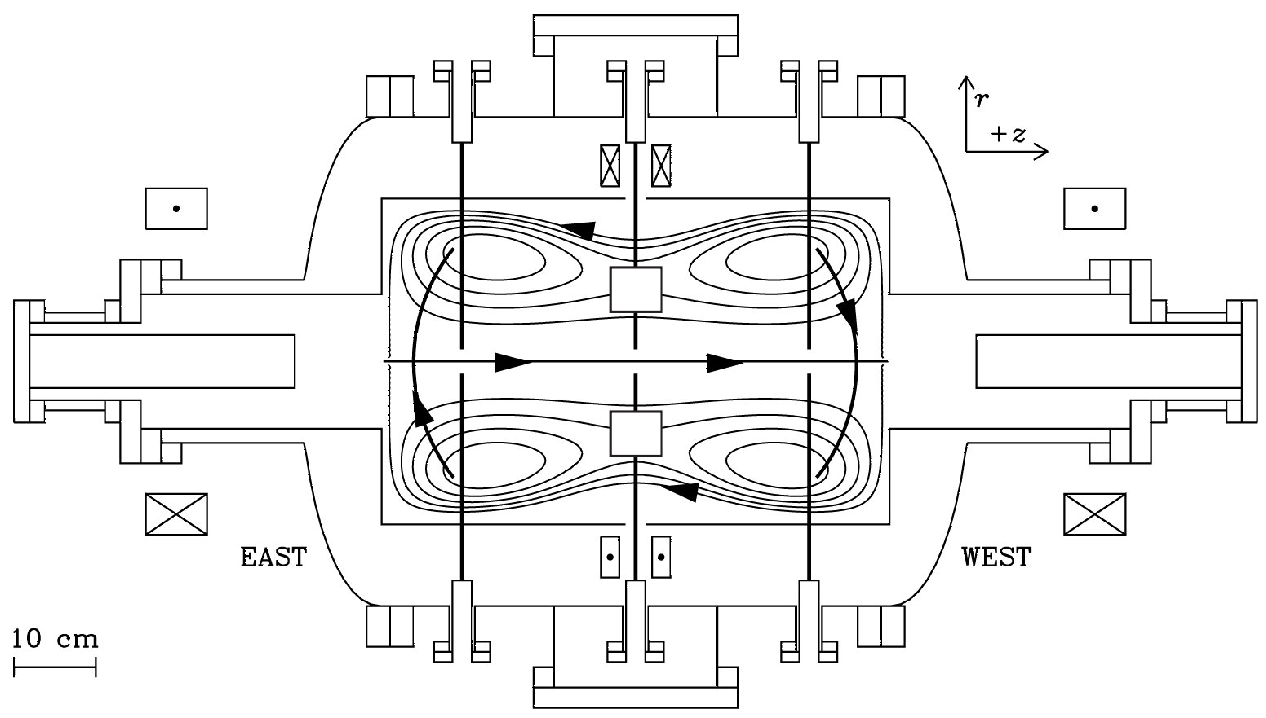

FIG. 1. Schematic of the SSX, shown in cross section. The device is cylindrically symmetric about the horizontal axis in the figure. Spheromak plasmas are formed in the guns on the east and west sides of the vacuum chamber and ejected into the main flux conserver. The lines of sight of the VUV monochromator, the SXR detector, and the ion Doppler spectrometer are at the midplane, aligned with the short axis of the chamber and perpendicular to the long axis, and here are indicated schematically by the white squares (see Fig. 9). Arrays of magnetic probes are shown schematically as six black lines in the figure. The probes are removed when we take spectroscopic data. The contours represent the magnetic field lines of a field-reversed configuration (FRC), the plasma structure that forms when two counterhelicity spheromaks merge.

has been studied with up to 600 individual internal magnetic probes operated simultaneously at $1.25 \mathrm{MHz}$ using a multiplexer system. ${ }^{31}$ Line averaged electron density is monitored with a quadrature $\mathrm{HeNe}$ laser interferometer. ${ }^{3}$ We can scan density in a range $n_{e}=1-10 \times 10^{14} \mathrm{~cm}^{-3}$ and operate at a typical value of $n_{e}=5 \times 10^{14} \mathrm{~cm}^{-3}$. The composition of the SSX plasma is primarily hydrogen, with trace concentrations of carbon and other impurities. The plasma is fully ionized and fully magnetized, with typical magnetic fields of $0.1 \mathrm{~T}$ and an ion gyroradius, $\rho_{i} \ll R$, where $R=0.2 \mathrm{~m}$ is the outer flux conserving boundary of the plasma (defined by a cylindrical copper wall). The Lundquist number $S$, the ratio of the resistive magnetic diffusion time $\tau_{R}$ to the Alfvén transit time $\tau_{A}$, is large for SSX, $S \approx 1000$. Accordingly, the global structure of SSX spheromaks is fully in the magnetohydrodynamic (MHD) regime $\left(S \gg 1, \rho_{i} \ll R\right)$.

In the experiments reported here, spectroscopic data are line integrated over a $1 \mathrm{~cm}$ wide chord through a diameter at the midplane with a time resolution of about $1 \mu \mathrm{s}$. In addition, an ensemble of 20-30 discharges are typically averaged for each measurement. Electron temperature and impurity concentration levels are inferred from model fits to data from a vacuum ultraviolet (VUV) spectrometer. Data are analyzed with a non-local thermodynamic equilibrium (non-LTE) excitation/ionization kinematics code. This modeling is also used to explore the extent to which time-dependent, nonequilibrium excitation and ionization has to be accounted for. Ion temperature is measured with an ion Doppler spectroscopy (IDS) instrument. ${ }^{32}$

In Sec. II, details of the spectral modeling using the PRISMSPECT code are presented. In Sec. III, determinations of impurity concentration from the VUV spectrometer are presented. In Sec. IV, we present measurements of the plasma electron temperature made using carbon emission line intensity ratios and compare these results with the C III ion temperature measured with IDS. In Sec. V, we discuss the progress of additional electron temperature measurements utilizing model fits to data from a four-channel soft $\mathrm{x}$-ray (SXR) array. A summary is presented in Sec. VI.

\section{SPECTRAL MODELING}

\section{A. PRISMSPECT}

We analyzed the SXR and VUV data using the PRISMSPECT non-LTE excitation/ionization code. ${ }^{33,34}$ Simulations were run for spatially uniform plasmas with a variety of temperatures, densities, and compositions. Model spectra and line strength ratios from these simulations were compared to spectroscopic data from SSX to determine best-fit values for the plasma parameters of interest. We also used timedependent simulations to study the plasma's approach to ionization/excitation equilibrium after spheromak formation.

PRISMSPECT models all significant radiative and collisional (including three-body) processes, although not charge exchange. The omission of charge exchange should not affect the models' relevance for comparison to spectroscopic data: the fractions of neutral hydrogen atoms and impurity ions in the SSX plasma are both low, so cold neutrals released from the walls during a discharge will almost always be ionized before they can undergo charge exchange with an impurity ion [the charge exchange mean free path of a C III ion, for example, is $l_{\mathrm{mfp}}>200 \mathrm{~m}$ (Ref. 35)]. In its timedependent mode, the PRISMSPECT code computes atomic level populations at each time step by integrating the relevant rate equations forward in time, and the calculated level populations and transition probabilities are used to produce a model spectrum. In the steady-state mode, equilibrium ionization balances and level populations are calculated by inverting a rate matrix. In both modes emissivities are calculated from the level populations and the emitted spectra are transported through the plasma taking optical depth effects into account. Doppler shifts from bulk motion are not accounted for in PRISMSPECT, but all relevant line-broadening mechanisms-including natural, Stark, and thermal broadening-are accounted for. ${ }^{36}$ This level of detail goes significantly beyond the coronal approximation, which we show below is necessary for accurate modeling of some of the emission lines we measure.

Atomic models for PRISMSPECT simulations are drawn 
from the ATBASE database. ${ }^{37}$ Many of the higher energy levels of impurity ions in the SSX plasma produce no strong spectral features, so we were able to make simplifications to the full ATBASE models and reduce simulation runtimes without sacrificing accuracy. Tests of a variety of atomic models revealed 200 energy levels per carbon ion and 50 levels per nitrogen ion to be sufficient, while the oxygen model used contained 50 levels for O III through O VII and one level for the other ions. Hydrogen in SSX is almost $100 \%$ ionized, and line radiation following recombination of protons and electrons was found to make only negligible contributions to SXR signals, so we used only the ground state of neutral hydrogen and ionized hydrogen in our atomic model for $\mathrm{H}$. For each element, adding more levels per ion stage beyond these described above did not lead to any significant change in the calculated spectra.

\section{B. Equilibration time}

The spheromak formation and expansion phases of an SSX discharge are characterized by rapidly changing temperatures and densities. ${ }^{5}$ Although it is difficult to estimate the time-varying conditions at the beginning of a discharge with a high degree of precision, we will demonstrate that the plasma is approximately in ionization and excitation equilibrium by the time it reaches the midplane. Thus, the time history of the spheromak plasma is not important, and its spectrum can be modeled with a simple collisional-radiative equilibrium approach, in which the density, temperature, and composition are the only important parameters.

The experiments we report on here focus primarily on the merging of two spheromaks rather than on the spheromak formation process itself. The merging and associated reconnection generally begins at about $t=30-40 \mu$ s and lasts for approximately $30 \mu \mathrm{s}$, after which the plasma achieves a quasisteady, relaxed configuration. The VUV monochromator and SXR view the plasma along fixed lines of sight through the midplane (see Fig. 1), so measured signals are typically zero for approximately the first $30 \mu \mathrm{s}$ of a discharge and peak around $t=50-60 \mu$ s. Steady-state simulations will therefore be sufficient if they can accurately describe the conditions in the plasma at $t>30 \mu \mathrm{s}$.

To test this, we calculated the evolution of the excitation and ionization of a hydrogen plasma with trace levels of carbon, nitrogen, and oxygen for a set of assumed density and temperature values as a function of time, based on our estimates of the conditions before, during, and after spheromak formation. A variety of initial conditions were tested, with electron temperatures in the plasma gun ranging from 0.025 to $60 \mathrm{eV}$. The ion density used was $8 \times 10^{15} \mathrm{~cm}^{-3}$ for the first $10 \mu$ s of the simulations (before the SSX plasma has left the gun), decreasing linearly to $5 \times 10^{14} \mathrm{~cm}^{-3}$ during the following $20 \mu$ s (modeling the plasma's expansion into the main flux conserver). We computed ultraviolet spectra as well as quantities such as the mean charge state at many times during these simulations, and saw that these tended to approach a steady state after $<10 \mu$ s. We then compared the spectra at late times, between 30 and $40 \mu \mathrm{s}$, with spectra calculated assuming equilibrium conditions. Indeed, these late-time spectra are nearly identical.

We can explore this numerical result analytically. Using the expression for the collisional excitation cross section, ${ }^{38}$ we find the mean excitation time in SSX with $n_{i}=5$ $\times 10^{14}$ ions $\mathrm{cm}^{-3}$ and $T_{e}=15 \mathrm{eV}$ to be $t \sim \sqrt{3 k T m_{e}^{3}} /\left(n \pi \hbar^{2}\right)$ $\sim 1.3 \times 10^{-7} \mathrm{~s}$. Therefore, in a period of several microseconds, each ion in SSX will be collisionally excited many times, indicating that equilibrium will be reached on a time scale $(<10 \mu \mathrm{s})$ that is short relative to the plasma lifetime, consistent with the results of the detailed simulations. Based on the observed insensitivity of late-time simulation results to initial conditions, tested for a range of initial temperatures and atomic level populations, we conclude that we can safely use steady-state simulations to model the VUV and SXR spectra.

\section{Line ratio modeling}

The SSX plasma, in the time leading up to reconnection, should be close to coronal equilibrium, in which all upward atomic transitions are assumed to be caused by collisions between ions and electrons, and all downward transitions are assumed to occur by spontaneous emission. Similarly, ionization is by electron impact and is assumed to be balanced by radiative recombination. Since collisional ionization and radiative recombination both scale with the square of the plasma density, the overall ionization balance in a coronal plasma is density independent, and the ratio of the intensities of two lines from different ionization stages of the same element can serve as an electron temperature diagnostic. The primary line ratio used for our temperature determinations was C III $97.7 \mathrm{~nm} / \mathrm{C}$ IV $155 \mathrm{~nm}$ (hereafter written as $\left.I_{97.7} / I_{155}\right)$. Representative spectra, calculated for two different temperatures, are shown in Fig. 2, where these two carbon lines are prominent.

Although the SSX plasma is close to coronal, we ran simulations that included the full range of atomic processes and found a noticeable density dependence in the $I_{97.7} / I_{155}$ ratio in the relevant density and temperature regimes, as shown in Fig. 3. Although the deviation from coronal equilibrium is slight, $\mathrm{C}$ III and $\mathrm{C}$ IV are subdominant ion stages at $T>10 \mathrm{eV}$ (see Fig. 4, where we show the temperaturedependent carbon ionization balance), so small absolute changes in their abundances can lead to large relative changes in the corresponding line intensity ratio. Because of this effect, it was necessary to obtain independent density measurements in order to use line ratios for precise calculations of electron temperature. We use an electron density value of $n_{e}=5 \times 10^{14} \mathrm{~cm}^{-3}$ based on measurements with the laser interferometer.

\section{IMPURITY DETERMINATIONS FROM VUV LINE MEASUREMENTS}

Individual emission line strengths are measured in SSX using a VUV monochromator with a focal length of $0.2 \mathrm{~m}$. Photons enter the device through a slit of adjustable width and strike a reflective diffraction grating, which selects and 

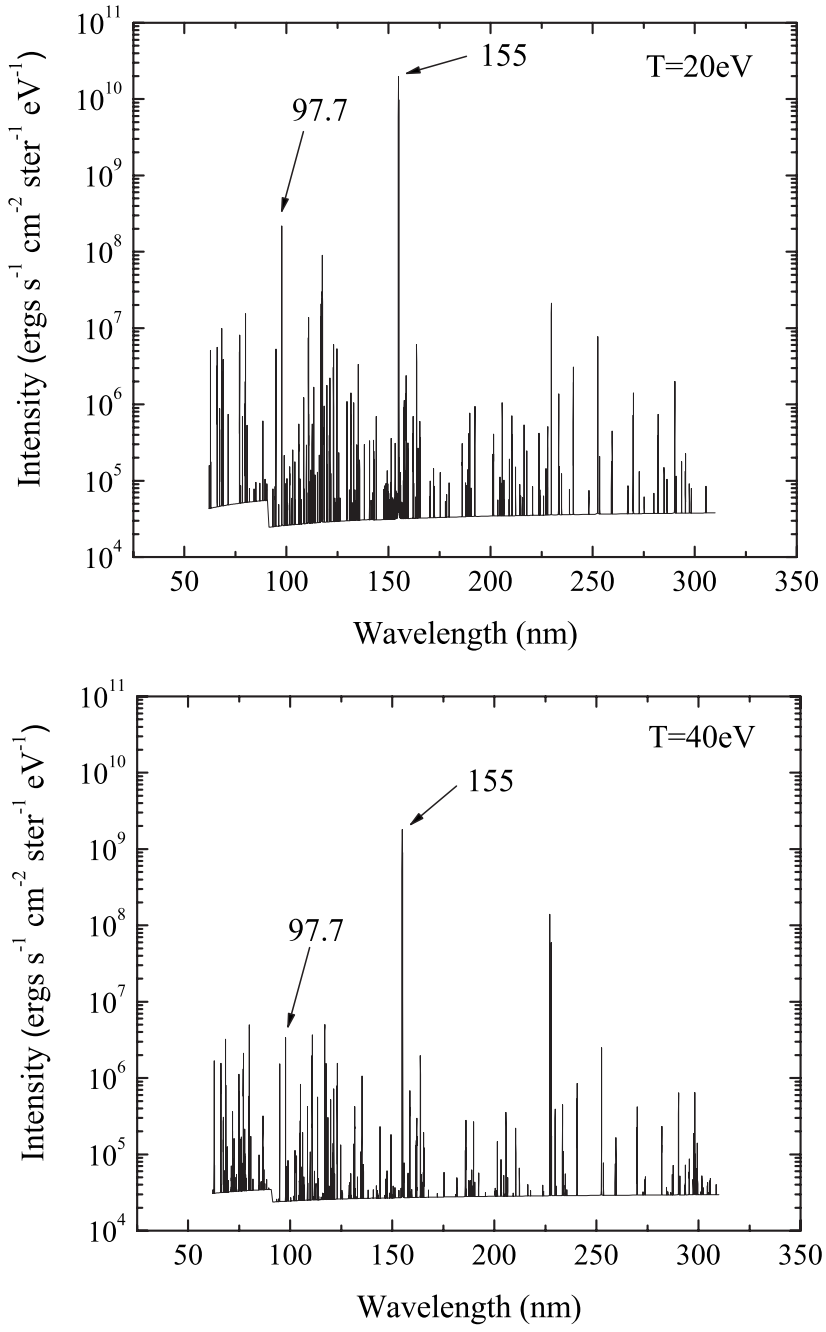

FIG. 2. Model spectra from steady-state simulations with $T_{e}=20 \mathrm{eV}$ (top) and $T_{e}=40 \mathrm{eV}$ (bottom). Both simulations assume a uniform $40 \mathrm{~cm}$ thick plasma composed of $99 \%$ hydrogen and $1 \%$ carbon with an ion density of $5 \times 10^{14} \mathrm{~cm}^{-3}$. The temperature dependence of the $I_{97.7} / I_{155}$ line ratio is evident. Note also the presence of the Lyman edge at $91.2 \mathrm{~nm}$, indicating that radiative recombination of hydrogen, rather than bremsstrahlung, is the dominant continuum process at work.

refocuses a narrow bandwidth around the desired central wavelength. Light leaving the diffraction grating is directed through an exit slit of adjustable width and into an $800 \mathrm{~V}$ photomultiplier tube (PMT). $500 \mu \mathrm{m}$ was found to be the optimal exit slit width, corresponding to a spectral resolution of $2 \mathrm{~nm}$. Signals from the PMT pass through a Stanford Research Systems SR570 current amplifier and are registered at $10 \mathrm{~ns}$ intervals by an oscilloscope and transferred to a computer using LABVIEW. Sample traces from individual discharges for two different lines are shown in Fig. 5.

PRISMSPECT was used to identify the carbon, nitrogen, and oxygen lines most likely to be strong in SSX, and we tuned the monochromator to measure these lines. The results are summarized in Table I. The wavelength-dependent sensitivity of the monochromator was estimated using measurements from the manufacturer of the normal incidence reflectivity of the $\mathrm{Al}+\mathrm{MgF}_{2}$ coating on the diffraction grating. Five impurity emission lines produced signals that could be reliably distinguished from background noise: C III $97.7 \mathrm{~nm}$,

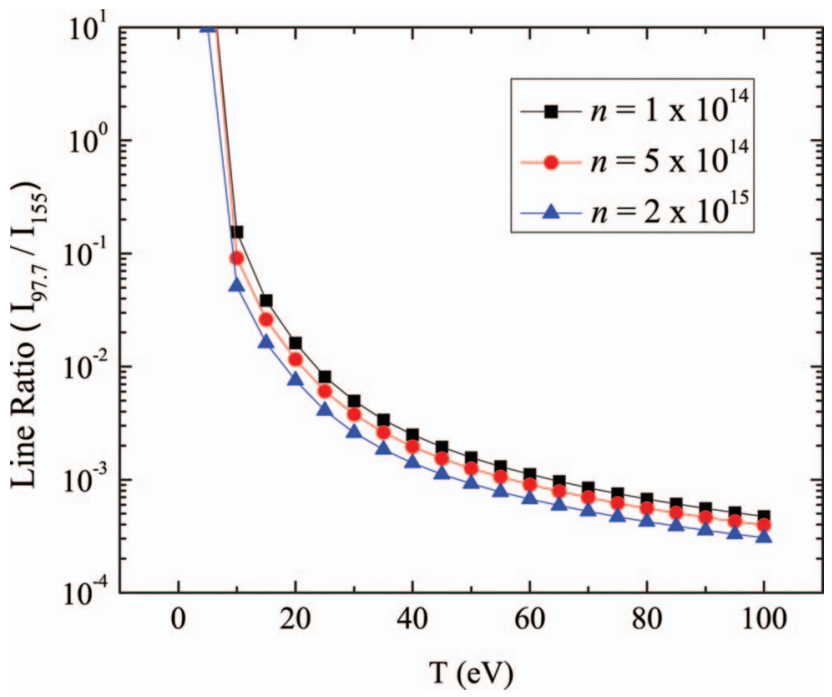

FIG. 3. (Color) Simulated $I_{97.7} / I_{155}$ line ratio plotted as a function of temperature for three different plasma densities (given in $\mathrm{cm}^{-3}$ ). Note the mild density dependence of this line ratio.

C IV $155 \mathrm{~nm}, \mathrm{C}$ III $229.7 \mathrm{~nm}$, N IV $124 \mathrm{~nm}$, and O v $63.0 \mathrm{~nm}$. The strongest of these was C IV $155 \mathrm{~nm}$, which consistently produced PMT currents over $1 \mathrm{~mA}$. C V is the dominant carbon ionization stage at SSX temperatures (see Fig. 4); however, being heliumlike, its resonance lines arise in the $K$-shell, and so are outside the wavelength range accessible to the monochromator. Therefore it is not necessarily surprising that no $\mathrm{C} v$ lines were detected. The $\mathrm{C} v$ line at $227 \mathrm{~nm}$ is from a transition between two excited levels, and so is not expected to be very strong.

The comparative strengths of $\mathrm{C}, \mathrm{N}$, and $\mathrm{O}$ lines can yield information about the relative fractions of these impurities present in SSX. These relative concentrations are a vital input for the simulations of SXR spectra to be discussed in Sec. V. Of course, line ratios involving $\mathrm{C}, \mathrm{N}$, and $\mathrm{O}$ depend on temperature, the main parameter we are trying to con-

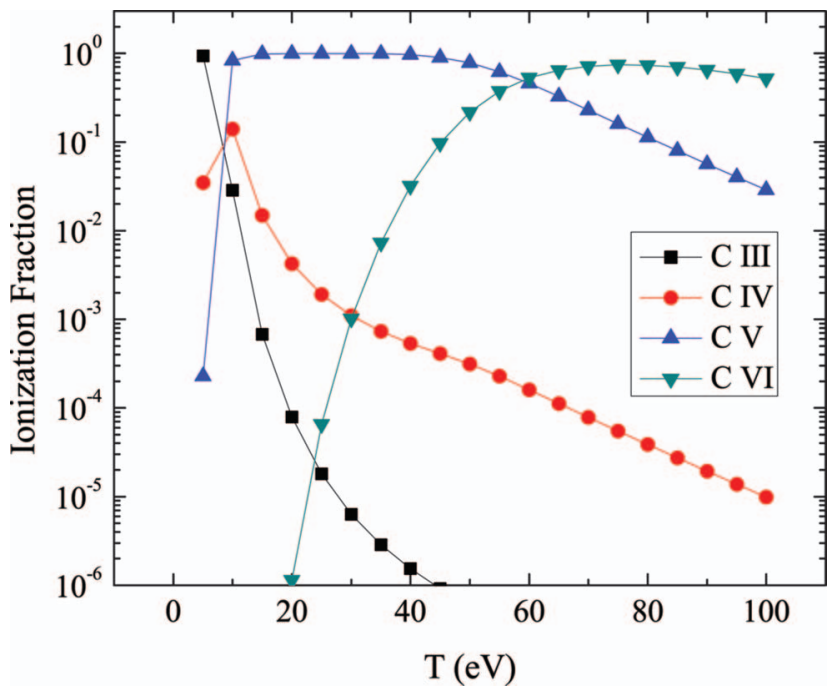

FIG. 4. (Color) Carbon ionization balance vs. electron temperature computed from PRISMSPECT simulations with an electron density of 5 $\times 10^{14} \mathrm{~cm}^{-3}$. 

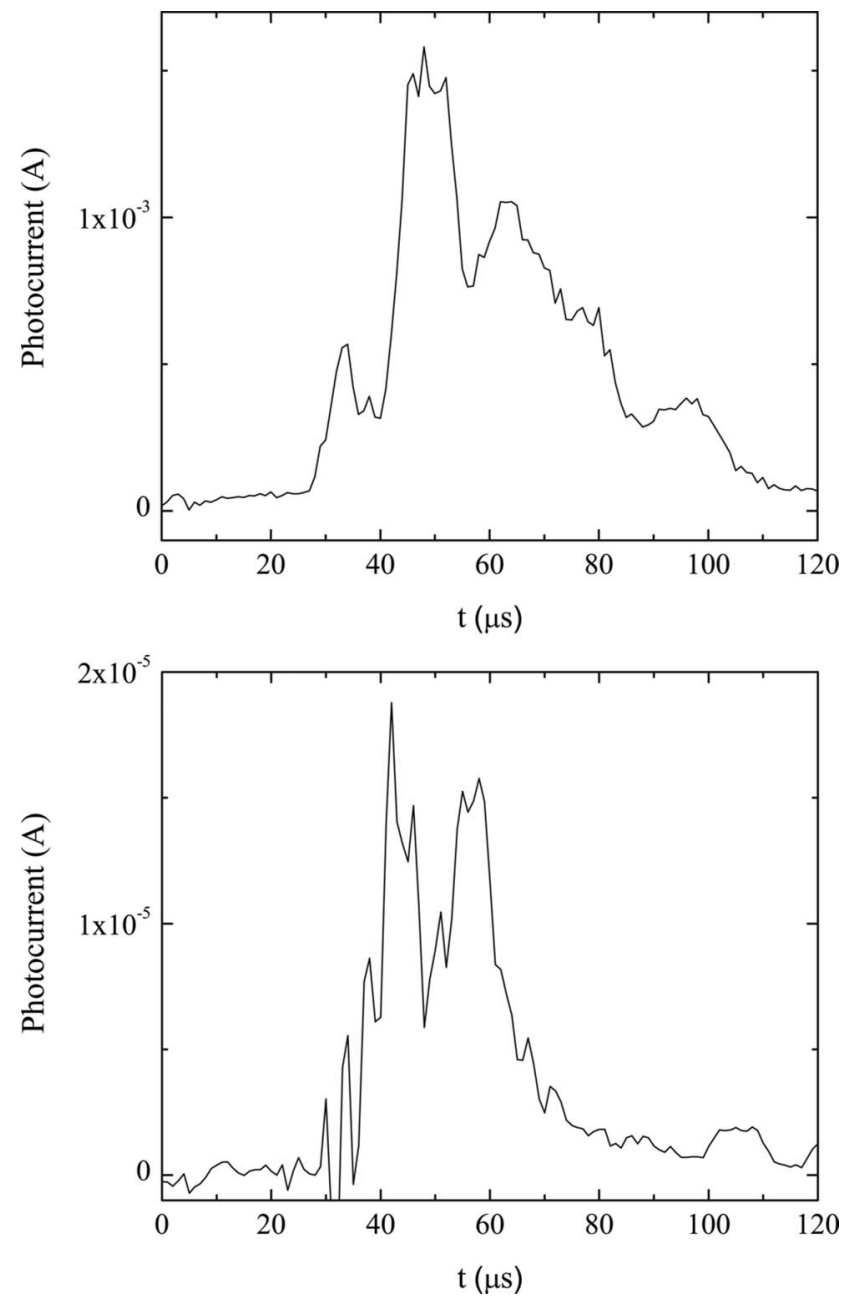

FIG. 5. Sample VUV monochromator data. Top: a measurement of the C IV $155 \mathrm{~nm}$ line intensity during a discharge with counterhelicity spheromak merging in SSX. Bottom: a measurement of the C III $97.7 \mathrm{~nm}$ line intensity during a single spheromak discharge.

TABLE I. Impurity emission lines measured with the VUV monochromator.

\begin{tabular}{lccc}
\hline \hline Ion & $\begin{array}{c}\lambda \\
(\mathrm{nm})\end{array}$ & Transition & Intensity $^{\mathrm{a}}$ \\
\hline $\mathrm{C}$ III & 97.7 & ${ }^{1} P_{1} \rightarrow{ }^{1} S_{0}$ & 10 \\
$\mathrm{C}$ IV & 155 & ${ }^{2} P_{1 / 2,3 / 2} \rightarrow{ }^{2} S_{1 / 2}$ & 350 \\
$\mathrm{C} \mathrm{V}$ & ${ }^{3} P_{0,1,2} \rightarrow{ }^{3} S_{1}$ & $\ldots$ \\
$\mathrm{C}$ III & 227.4 & ${ }^{1} D_{2} \rightarrow{ }^{1} P_{1}$ & 2 \\
$\mathrm{~N}$ IV & 229.7 & ${ }^{1} P_{1} \rightarrow{ }^{1} S_{0}$ & $\ldots$ \\
$\mathrm{N} \mathrm{v}$ & 76.5 & ${ }^{2} P_{1 / 2,3 / 2} \rightarrow{ }^{2} S_{1 / 2}$ & 25 \\
O IV & 124 & ${ }^{2} P_{1 / 2,3 / 2} \rightarrow{ }^{2} P_{1 / 2,3 / 2}$ & $\ldots$ \\
O v & 55.4 & ${ }^{1} P_{1} \rightarrow{ }^{1} S_{0}$ & 15 \\
O IV & 63.0 & ${ }^{2} D_{3 / 2,5 / 2} \rightarrow{ }^{2} P_{1 / 2,3 / 2}$ & $\ldots$ \\
O VI & 78.9 & ${ }^{2} P_{1 / 2,3 / 2} \rightarrow{ }^{2} S_{1 / 2}$ & $\ldots$ \\
\hline \hline
\end{tabular}

${ }^{a}$ Relative intensities quoted are averaged over the 40-60 $\mu$ s interval of each discharge. The signals are scaled based on our estimate of the wavelength-dependent calibration of the monochromator and normalized so that a value of 1 corresponds to the weakest signal we can readily distinguish from the background noise.
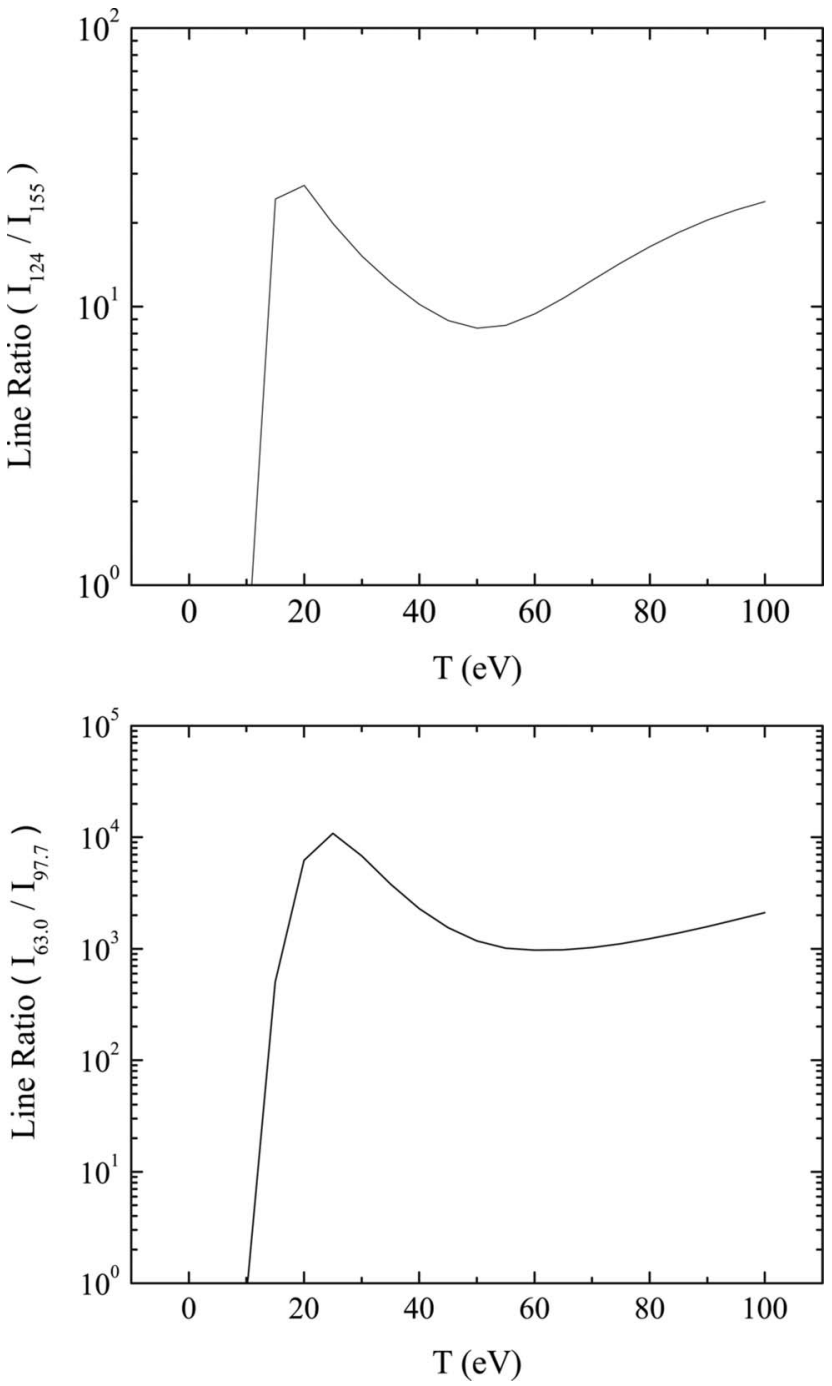

FIG. 6. Line ratios calculated from PRISMSPECT simulations with $0.1 \%$ carbon, nitrogen, and oxygen impurities and $n_{i}=5 \times 10^{14} \mathrm{~cm}^{-3}$. The N v 124 $\mathrm{nm}$ line is predicted to be at least eight times stronger than the C IV $155 \mathrm{~nm}$ line for plausible SSX temperatures (top), and the $\mathrm{OV} 63.0 \mathrm{~nm}$ line is predicted to be over 500 times stronger than the C III $97.7 \mathrm{~nm}$ line (bottom). The measured strength of the carbon lines relative to the others is much higher than these predictions, allowing us to estimate the concentrations of nitrogen and oxygen relative to carbon in the SSX plasma.

strain. Nevertheless, estimates of relative impurity concentrations can be made through comparisons of VUV line measurements with simulations.

We consider the ratios N v $124 \mathrm{~nm} / \mathrm{C}$ IV $155 \mathrm{~nm}$ and O v $63.0 \mathrm{~nm} / \mathrm{C}$ III $97.7 \mathrm{~nm}$, in order to constrain the concentrations of $\mathrm{N}$ and $\mathrm{O}$ relative to $\mathrm{C}$. We use the different carbon lines in order to minimize the temperature dependence of each diagnostic line ratio. As shown in Fig. 6, with equal concentrations of $\mathrm{C}, \mathrm{N}$, and $\mathrm{O}$ present, the simulated $I_{124} / I_{155}$ ratio is $>8 / 1$ for all plausible SSX plasma temperatures and about $25 / 1$ for $T_{e}=15 \mathrm{eV}$. Yet, the measured intensity ratio is $1 / 14$, implying a concentration of $\mathrm{N}$ that is roughly 350 times lower than that of C. Similar modeling for oxygen shows that the $I_{63.0} / I_{97.7}$ ratio is $\sim 700 / 1$ at $15 \mathrm{eV}$; however, the measured intensity ratio is $3 / 2$, implying that carbon is $\sim 500$ times more abundant than oxygen in SSX (we have adopted $15 \mathrm{eV}$ as a typical temperature based on the results described 
in Sec. IV). The low concentrations of $\mathrm{N}$ and $\mathrm{O}$ are not surprising, as copper gasket seals are used on the SSX device and we employ an intensive program of high temperature bakeout and helium glow discharge conditioning before running the experiment.

Some insight into absolute impurity concentrations in the plasma can be garnered from an analogous analysis of the H I $121.6 \mathrm{~nm} / \mathrm{C}$ III $97.7 \mathrm{~nm}$ line ratio, measured to be $\sim 1 / 2$ in SSX. Simulations with $99 \% \mathrm{H}$ and $1 \% \mathrm{C}$ (using the full ATBASE hydrogen model) predict a line ratio of $1 / 2$ at 20 $\mathrm{eV}$, so $1 \%$ carbon concentration is a reasonable estimate for SSX, although edge effects would have to be considered for a more precise determination.

\section{TEMPERATURE MEASUREMENTS}

\section{A. Electron temperature}

During the merging of two spheromaks, reconnection converts stored magnetic energy into thermal and kinetic energy. Understanding the details of this energy budget is vital for modeling coronal heating and other astrophysical phenomena. Electron temperatures during counterhelicity spheromak merging were calculated by comparing measured and simulated (Fig. 3) values of the carbon line ratio $I_{97.7} / I_{155}$. We take resonance scattering into account, but the lines are optically thin, so the comparison is quite straightforward. We determine an average electron temperature at each $1 \mu$ s bin from the ratio of the mean line intensities calculated from a set of 25 discharges for each line. The standard deviation of the line ratio at each time was used to define the uncertainty in the electron temperature. The results of the analysis of the $I_{97.7} / I_{155}$ ratio measurements are shown in Fig. 7.

The average electron temperature during counterhelicity discharges increases from 12 to $19 \mathrm{eV}$ during the 35-70 $\mu \mathrm{s}$ time period (reconnection begins to occur by $t \sim 30-40 \mu \mathrm{s}$ ), providing substantial evidence for magnetic energy being converted into thermal energy. A lesser temperature increase $(\sim 2 \mathrm{eV})$ is seen during single spheromak discharges, as the plasma relaxes into a lower-energy configuration around $t$ $=50 \mu \mathrm{s}$. This measurement provides something of a control, indicating that the heating observed during reconnection is significant.

\section{B. Ion temperature}

The temperature of carbon ion impurities was measured by Doppler spectroscopy. The IDS instrument developed for SSX has sufficient time resolution (approximately $1 \mu \mathrm{s}$ ) to follow the MHD time scale evolution of the plasma, and sufficient spectral resolution $\left(\delta \lambda / \lambda \approx 2 \times 10^{-5}\right)$ to measure carbon ion temperatures down to approximately $3.4 \mathrm{eV}$ and Doppler shifts of as little as $6 \mathrm{~km} / \mathrm{s}$. The view chord of the IDS is very narrow $(f / \# \approx 100)$, and its $15 \mathrm{~mm}$ diameter is small compared to the size of the plasma; the IDS therefore performs a spatial line integration to a very good approximation. The simple interpretation of the measured line shapes as a Doppler shifted and broadened spectral line is often complicated significantly by the effects of the flow profile along
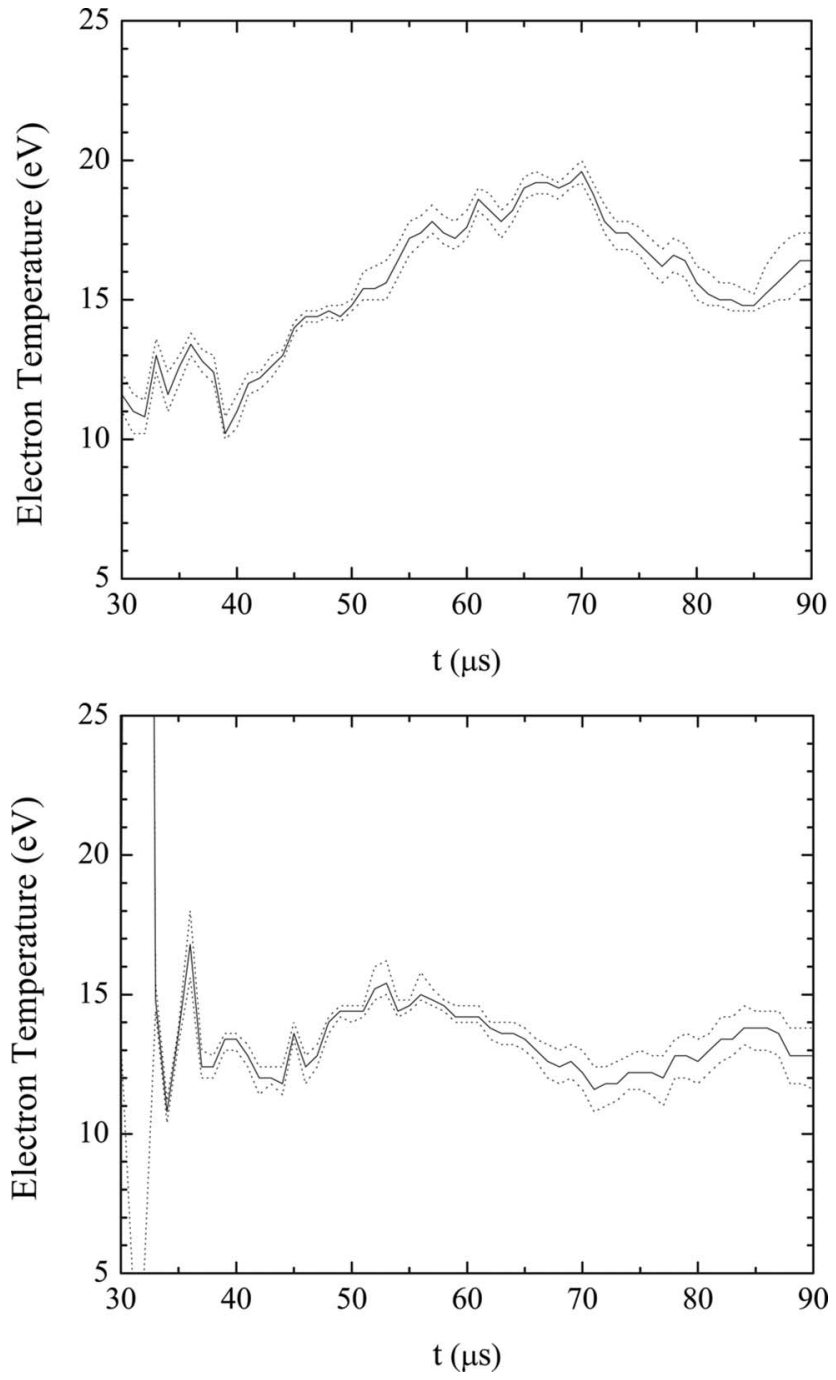

FIG. 7. Electron temperatures for counterhelicity merging (top) and single spheromak discharges (bottom) derived from $I_{97.7} / I_{155}$ measurements averaged over 25 discharges for each line. An ion density of $5 \times 10^{14} \mathrm{~cm}^{-3}$ was assumed. Dashed lines give the uncertainty range, defined as the temperatures implied by a line ratio one standard deviation above or below the mean.

the line of sight. For example, the line width generated by a flow varying by just $\pm 10 \mathrm{~km} / \mathrm{s}$ (about an order of magnitude less than the Alfvén speed) along the IDS line of sight is equivalent to a thermal width of about $50 \mathrm{eV}$.

The carbon ion temperature data from the IDS are presented in Fig. 8. This measurement was made with the IDS collecting C III emission at $229.687 \mathrm{~nm}$ along a diameter of the plasma at the midplane, and is the average of ten spheromak merging experiments. Line shapes showing evidence of nonthermal broadening or small signal strength were eliminated from the average; this occurred most often at times earlier than $40 \mu \mathrm{s}$. The ion temperature during both counterhelicity merging and single spheromak discharges was observed to increase from approximately 13 to $22 \mathrm{eV}$ during the time interval from 40 to $55 \mu \mathrm{s}$.

\section{Resistive heating interpretation}

The similar ion temperatures observed during counterhelicity merging and single spheromak discharges (Fig. 8) indicate that most of the observed ion heating can be attributed 

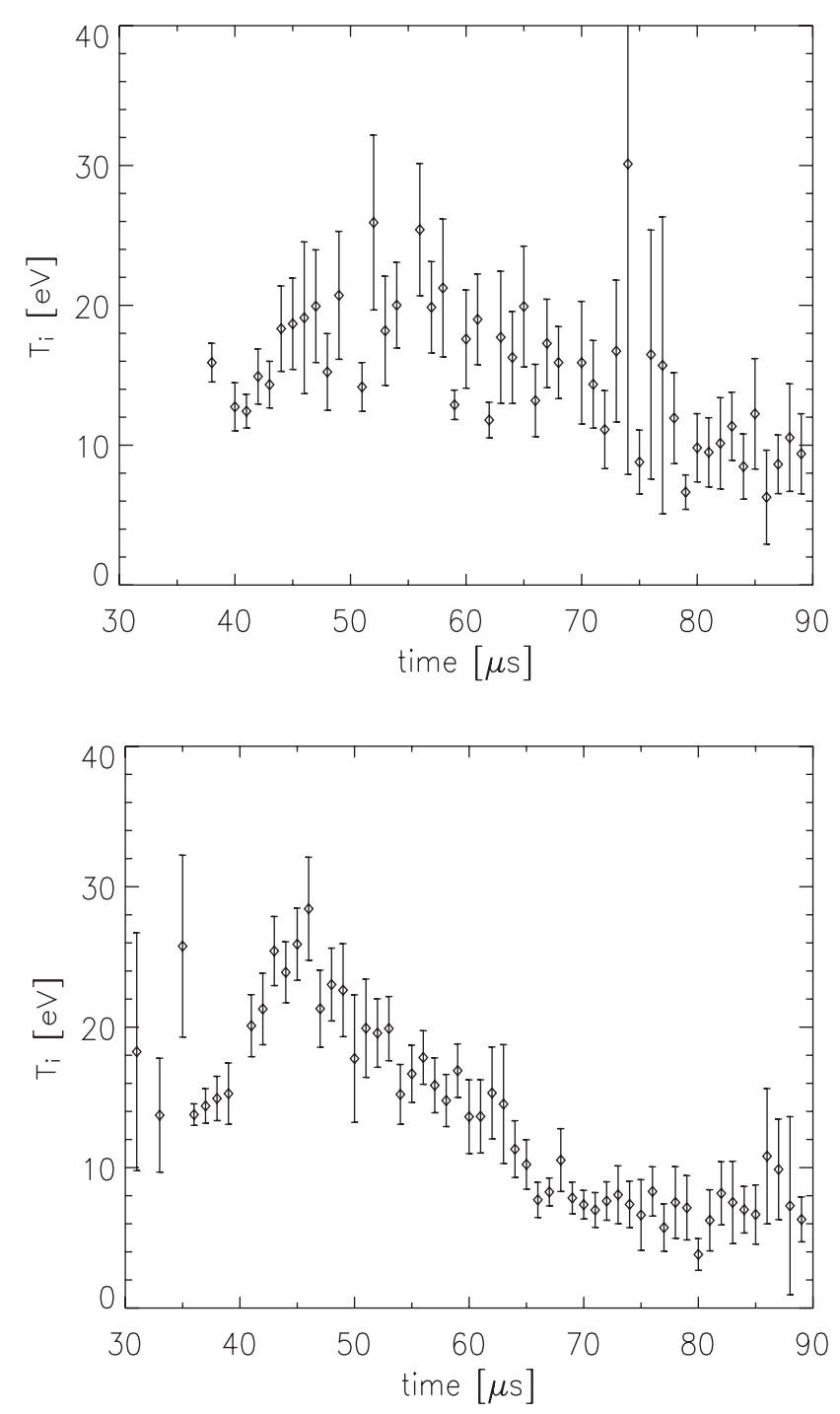

FIG. 8. Thermal Doppler broadening of the C III $229.687 \mathrm{~nm}$ line observed with the IDS instrument viewing along a midplane diameter of the plasma during counterhelicity merging (top) and single spheromak discharges (bottom). Each data point is the average of up to ten discharges; data with small signal strength or showing nonthermal (flow profile) effects were dropped from the average.

to spheromak relaxation and accompanying small-scale magnetic reconnection events throughout the plasma volume, rather than to large-scale reconnection during merging at the midplane. Likewise, some electron heating is observed during the $40-55 \mu$ s period of single spheromak discharges. At $t=70 \mu \mathrm{s}, T_{e}$ during counterhelicity discharges reaches a maximum of $\sim 19 \mathrm{eV}$, while $T_{e}$ for single spheromak discharges is $\sim 13 \mathrm{eV}$. Assuming similar cooling rates for the two situations, we can attribute the $6 \mathrm{eV}$ difference to energy input from magnetic reconnection during spheromak merging.

At a density of $5 \times 10^{14} \mathrm{~cm}^{-3}$, a $6 \mathrm{eV}$ increase in $T_{e}$ across the $0.075 \mathrm{~m}^{3}$ plasma volume corresponds to a thermal energy input of $36 \mathrm{~J}$ to the electrons. Earlier measurements of the magnetic flux and reconnection rate ${ }^{6}$ in SSX imply a reconnection electric field of $E \approx 100 \mathrm{~V} / \mathrm{m}$. Thus the power input through Joule heating in the reconnection region is given by $P / V=E^{2} / \eta$, where the Spitzer resistivity at $15 \mathrm{eV}$ is

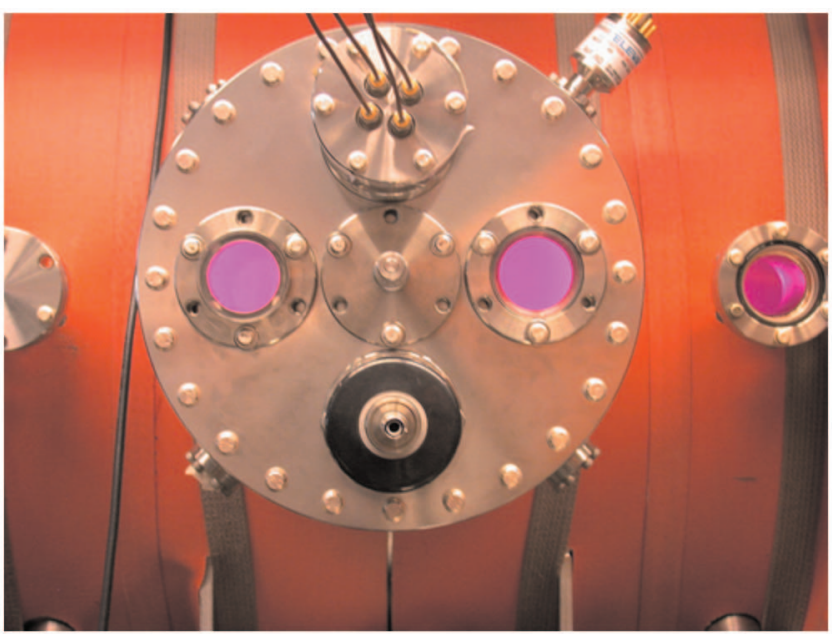

FIG. 9. (Color) Location of SXR at the midplane on the SSX machine (see the schematic in Fig. 1). The four wires at the top of the image carry current from the SXR photodiodes. Clockwise from upper left, the diodes are filtered by foils made of Al, Zr, Sn, and Ti. The port for the IDS is visible at the bottom center of this image. Note that hydrogen Balmer series recombination emission is visible through the windows in the vessel in this photograph.

$\eta \approx 8.9 \times 10^{-6} \Omega \mathrm{m}$. Magnetic data suggests that reconnection occurs primarily within an annulus with inner radius of $\sim 11 \mathrm{~cm}$, outer radius of $\sim 17 \mathrm{~cm}$, and thickness of $\sim 1 \mathrm{~cm} .{ }^{6,39}$ Thus, for reconnection at the midplane lasting $35 \mu \mathrm{s}$, the total resistive power dissipation is $\sim 21 \mathrm{~J}$.

Based the agreement to within a factor of 2 between the observed thermal energy increase and the expected resistive power dissipation, we can conclude that the magnetic and spectroscopic data provide a self-consistent picture of the energy balance during spheromak merging at SSX. Our simple analysis makes no attempt to account for spatially varying densities and temperatures within the plasma, nor is the size of the reconnection region well constrained by the magnetic data. Thus, we need not conclude that a stronger dissipative mechanism than classical resistivity is at play during the reconnection process. More sophisticated simulations are underway to further our understanding of this issue.

\section{SXR MEASUREMENTS}

The SSX SXR detector, $6,11,19,40-42$ mounted at the midplane (see Fig. 9) and sampling a chord of plasma in the reconnection region, measures the intensity of EUV and SXR emission from the SSX plasma with a time resolution better than $10 \mathrm{~ns}$. The SXR consists of a matched set of four International Radiation Detectors AXUV silicon $p-n$ junction photodiodes filtered by thin films of aluminum $(100 \mathrm{~nm}$ thick), titanium $(50 \mathrm{~nm})$, tin $(100 \mathrm{~nm})$, and zirconium (100 $\mathrm{nm})$. The variation in the spectral response functions of the filters at EUV and SXR energies, shown in Fig. 10, allows us to garner information not just about the time-dependent intensity of the SXR emission, but also about broad spectral properties of the emission. Ultimately, we intend to use this data to supplement the VUV line ratio analysis and independently derive the electron temperature in SSX. Although the analysis of the SXR data lacks the simplicity of the line ratio 

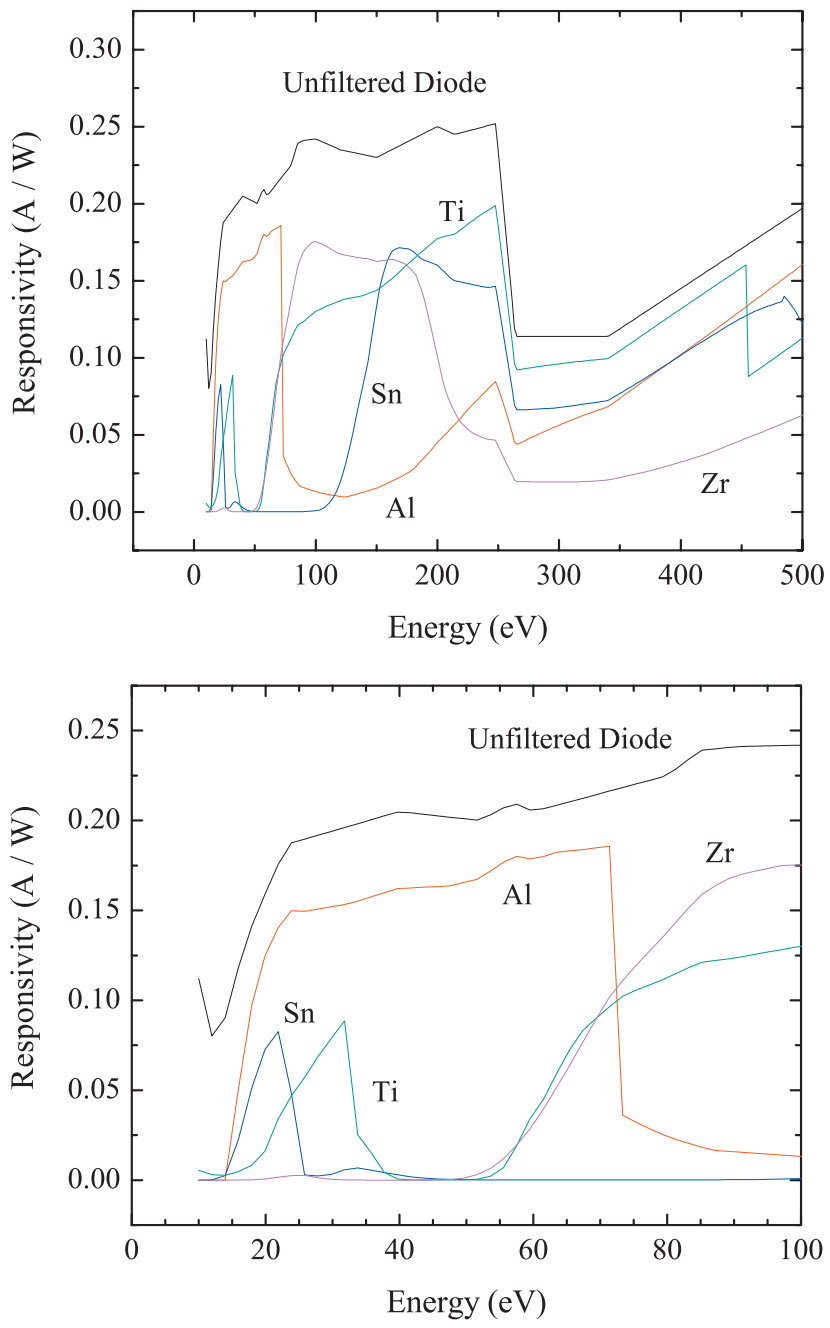

FIG. 10. (Color) SXR filter responsivities (Refs. 43 and 44). The colored lines show the response function of the filtered diodes in the $10-500 \mathrm{eV}$ range, and the black line shows the response function of the unfiltered diode. Line emission at $E>500 \mathrm{eV}$ was negligible at all simulation temperatures. The lower panel shows the same responsivity curves, but zoomed in on the range below $100 \mathrm{eV}$, where the bulk of the plasma emission is expected.

measurement we make with the VUV monochromator, it has the distinct advantage of allowing us to determine electron temperatures for individual discharges since the flux through all four filters can be measured simultaneously. In addition, the SXR is sensitive to significantly higher energy photons than the VUV monochrometer, and thus can potentially measure emission from hotter plasma and even emission produced by a possible high-energy, non-Maxwellian component of the electron population.

In Fig. 11 we show the time-dependent SXR signals measured in the four different SXR filters during one counterhelicity spheromak merging shot. X-ray emission is first detected when the plasma reaches the midplane at 25-30 $\mu \mathrm{s}$ and ramps up quite rapidly at 35-40 $\mu$ s following the onset of magnetic reconnection. Intense emission is sustained for several tens of microseconds, with the relaxation to a merged, equilibrium configuration typically around $60 \mu \mathrm{s}$ corresponding to a strong decrease in the SXR signal, as we expect for thermal emission arising from the heating associated with reconnection. We note that the signal in the $\mathrm{Al}$

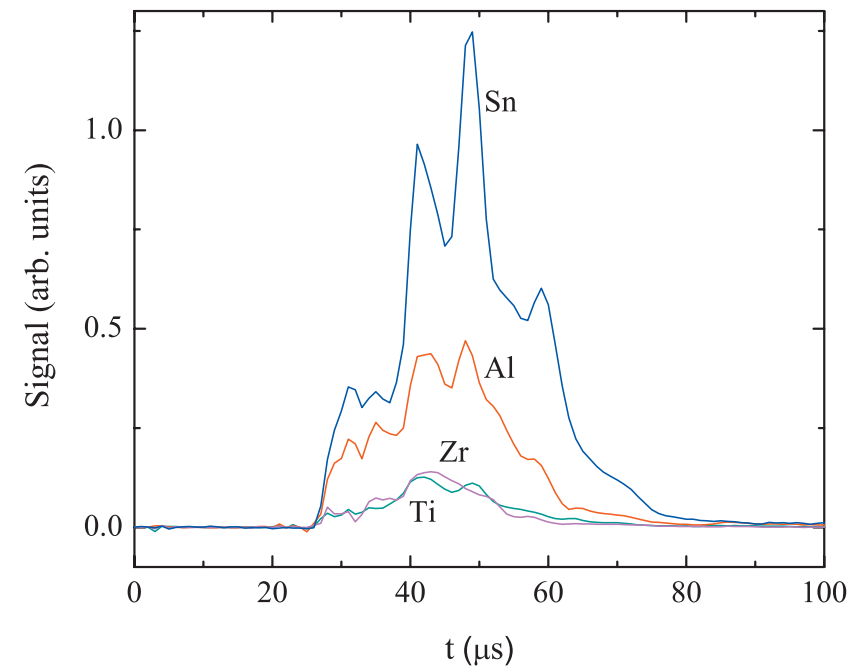

FIG. 11. (Color) SXR data from a discharge with counterhelicity merging, smoothed over $1 \mu$ s intervals. Note the onset of strong SXR emission around $30-40 \mu$ s, when reconnection begins.

bandpass is stronger than that seen in $\mathrm{Ti}$ or $\mathrm{Zr}$, which is not surprising given the higher and broader spectral responsivity of the Al channel, shown in Fig. 10. The very strong Sn channel signal is quite unexpected, however, as the responsivity of this channel is not the highest of the four filters at any photon energy. We verified that there is no low-energy leak (below $8 \mathrm{eV}$ ) by blocking this channel with UV fused silica and sapphire filters. However, there must be a problem with this channel, as there is no physical basis for the relative signal being this high, so we exclude the Sn channel data from the analysis we describe below.

In Fig. 12 we again show the SXR filter traces, but at an expanded scale afforded by the elimination of the $\mathrm{Sn}$ data. The time dependence of each signal appears similar, but in the lower panel, where we show the time-dependent channel intensity ratios, it is clear that the spectral signal of the $\mathrm{x}$-ray emission changes in the interval $35 \mu \mathrm{s}<t<60 \mu \mathrm{s}$. This is exactly the time frame of reconnection and relaxation toward a magnetic equilibrium that is seen in the magnetic data. The $\mathrm{Ti} / \mathrm{Al}$ ratio is the most constant of the three during this time; the changes in the spectral energy distribution seem to be primarily due to the $\mathrm{Zr}$ signal. Looking at the response curves (Fig. 10), Zr has a significantly lower spectral response than $\mathrm{Al}$ and $\mathrm{Ti}$ at photon energies greater than $300 \mathrm{eV}$ and a relatively high response at lower energies. Since the change seen in the channel intensity ratios with time (lower panel of Fig. 12) is in the sense of the $\mathrm{Zr}$ signal becoming weaker faster than the signal in the other two channels, this implies a spectral hardening with time during reconnection.

We model the SXR emission from the SSX plasma using the same type of PRISMSPECT simulations we described earlier in the context of the VUV line ratio modeling. For the $\mathrm{SXR}$ analysis, we include $\mathrm{C}, \mathrm{N}$, and $\mathrm{O}$ in the ratios determined from the VUV line intensity measurements, and perform similar non-LTE, steady-state simulations, calculating the emergent spectrum as a function of temperature at high resolution between 10 and $1000 \mathrm{eV}$. The absolute impurity concentrations (relative to hydrogen) are not important as 

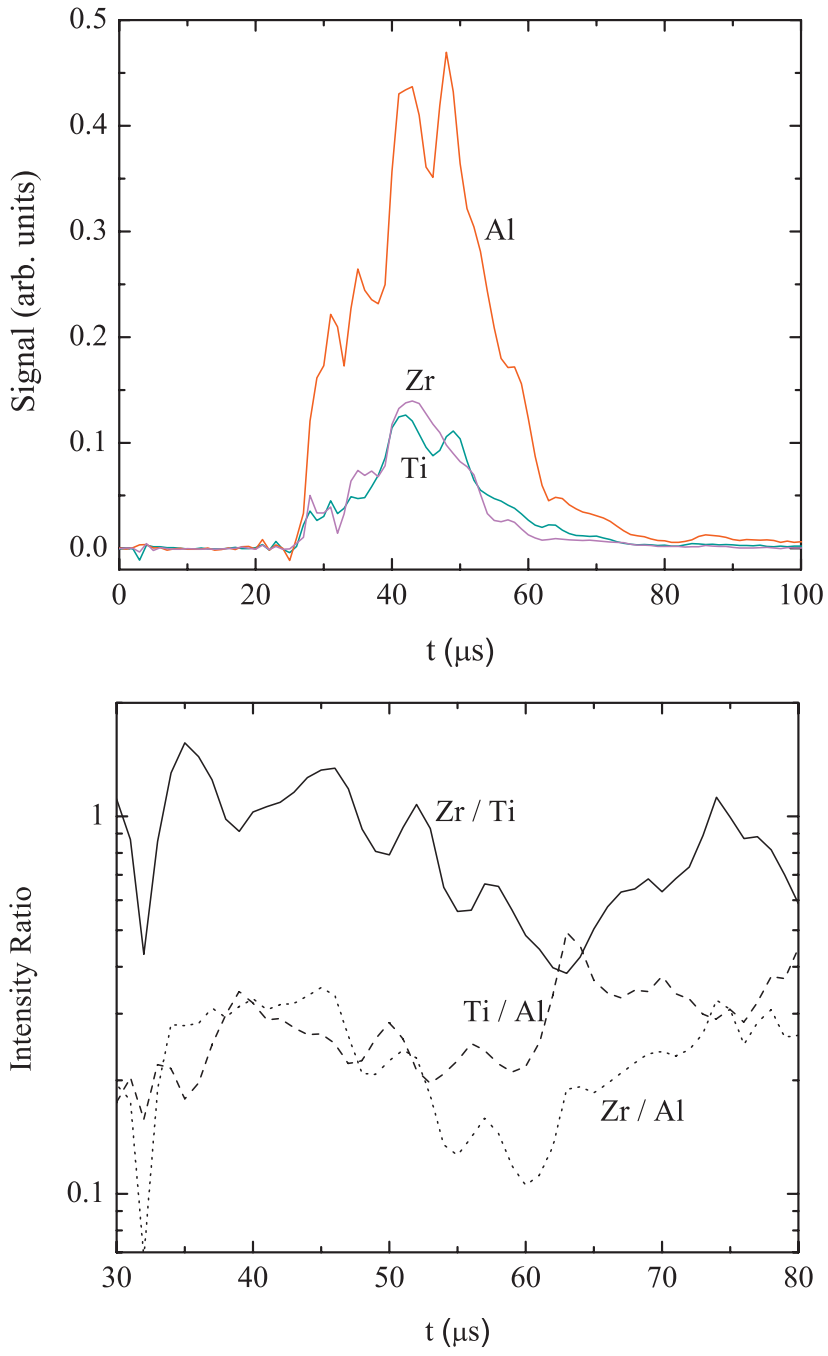

FIG. 12. (Color) Top: SXR data for the Al, Zr, and Ti filters from a discharge with counterhelicity merging, smoothed over $1 \mu$ s intervals. Bottom: time evolution of filter ratios for the discharge. Sn filter data are omitted in order to more clearly show the time evolution of the other three ratios.

long as the carbon concentration is above one part in $10^{4}$, as our simulations show that the spectra are completely dominated by impurity line emission even at low concentration. A representative model spectrum is shown in Fig. 13. We multiply the temperature-dependent model SXR spectra by the filter responsivities and integrate over photon energy to produce a model of the relative signal strength in each of the three ( $\mathrm{Zn}, \mathrm{Al}$, and $\mathrm{Ti}$ ) channels. We then compare the measured intensity ratios from the three channels to the temperature-dependent model predictions. Note that by using ratios of the intensities we avoid errors due to our uncertain knowledge of the exact emissivity in the volume of plasma sampled by the SXR (which is affected by the detailed density distribution in the sampled volume, as well as the absolute impurity concentrations).

By comparing these model channel intensity ratios to the measured ratios in $1 \mu$ s intervals, we can, in principle, determine the time evolution of the electron temperature during a single discharge. The model-data comparison procedure for two time snapshots is shown in Fig. 14. What we find, however, is that there are times (as in the bottom panel in the

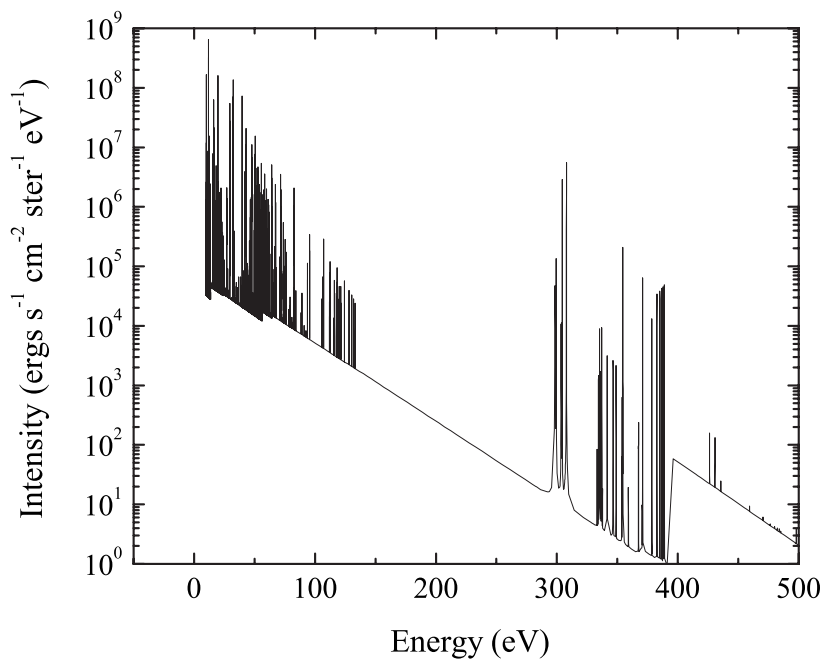

FIG. 13. Model spectrum from a steady-state simulation with $T_{e}=30 \mathrm{eV}$ and $n_{i}=5 \times 10^{14} \mathrm{~cm}^{-3}$. Impurity concentrations of $1 \%$ carbon, $0.002 \%$ oxygen, and $0.003 \%$ nitrogen were included. The plasma emits primarily at $E$ $<150 \mathrm{eV}$, but SXR will also measure substantial emission from the $\mathrm{C} \mathrm{V}$ and $\mathrm{C}$ VI resonance lines between 300 and $400 \mathrm{eV}$. Note that the $\mathrm{Zr}$ channel has the lowest responsivity at these energies, which is counter to what is seen below $100 \mathrm{eV}$.

figure) where no single temperature or small range of temperatures can explain all three intensity ratios. Perhaps the emission at high energies is sensitive to some nonequilibrium physics that we do not include in our models. We have modeled the inclusion of $\mathrm{Cu}$ and other possible impurities, but thermal line emission from additional species does not appear to explain the discrepancies.

\section{SUMMARY}

We have shown that high time resolution UV spectroscopy is a useful tool for characterizing the physical properties of the SSX plasma during and after magnetic reconnection. Electron temperature can be estimated from impurity emission line ratios, but it is important to take into account deviations from coronal equilibrium, which requires detailed spectral modeling. This same modeling also shows that ionization and excitation equilibrium is reached within $10 \mu \mathrm{s}$ after the plasma is discharged from the guns so that steadystate simulations are sufficient for describing the plasma emission at the time that reconnection occurs (beginning 30 or $40 \mu$ s after the plasma discharge) and, furthermore, that the exact conditions in the plasma guns do not need to be known in order to accurately model the plasma properties around the time of reconnection.

Comparing model line ratios with measured UV line emission from various impurity species allows us to place constraints on the relative impurity concentrations in the plasma. Carbon is the dominant impurity in SSX, with a concentration approximately 350 times greater than that of nitrogen and 500 times greater than that of oxygen. However, even the less abundant elements make a significant contribution to the UV and SXR line emission.

For the first time in SSX we have made precise, high time resolution measurements of the electron temperature 

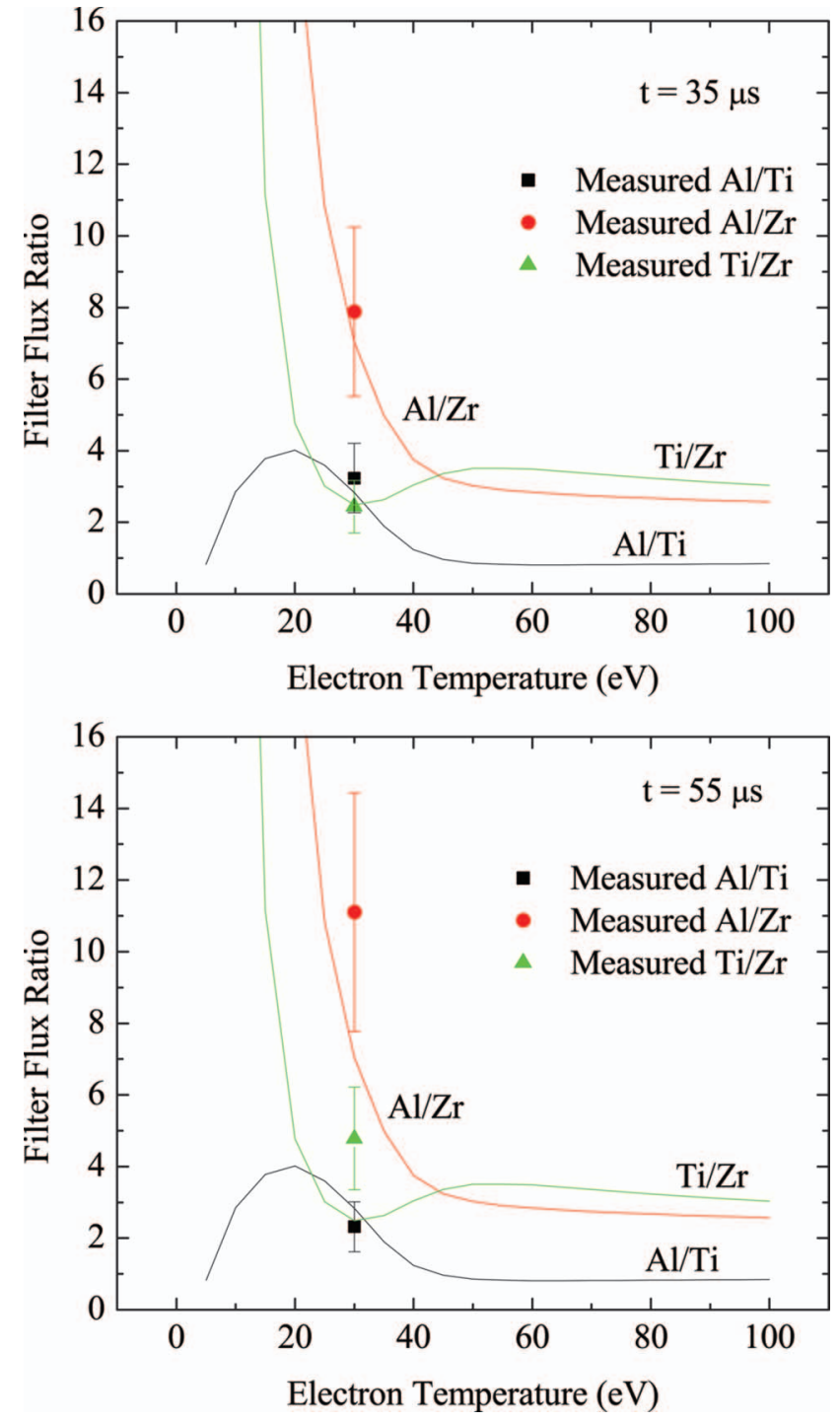

FIG. 14. (Color) Illustration of the process used to fit SXR data to model spectra and determine $T_{e}$. Channel intensity ratios calculated from models are plotted as a function of temperature (solid lines). Measured ratios at $t$ $=35 \mu$ s (top panel) and $t=55 \mu$ s (bottom panel) during a typical single spheromak discharge are plotted as points at the location of the calculated best-fit temperature for the time step. For illustrative purposes, 30\% error in the measured ratios is assumed. At $t=35 \mu \mathrm{s}$, a good fit is achieved at $T$ $=30 \mathrm{eV}$. However, at $t=55 \mu \mathrm{s}$, all three measured ratios cannot match the models at any one temperature, and the best-fit temperature of $30 \mathrm{eV}$ may not accurately reflect the actual $T_{e}$ in the plasma.

during counterhelicity spheromak merging. Energy released by magnetic reconnection causes $T_{e}$ to rise from approximately 12 to $19 \mathrm{eV}$ on average during the $35-70 \mu$ s interval of a discharge. A smaller increase in $T_{e}$ is observed during single spheromak discharges. Mean ion temperatures, as determined by line width measurements using an ion Doppler spectrometer, also rise from approximately 13 to $22 \mathrm{eV}$ during the 40-55 $\mu$ s interval of a discharge, but the increase in $T_{i}$ is similar for shots with and without spheromak merging and thus must be largely unrelated to reconnection at the midplane. We propose a simple resistive heating model for the conversion of magnetic energy to thermal energy during spheromak merging and find reasonable agreement between previously published magnetic data and the new spectroscopic measurements.

Utilizing the information about impurity concentrations derived from UV line intensity measurements, we have developed a framework for monitoring electron temperature during individual discharges using a broadband SXR detector, and the same spectral modeling tools that we used successfully to model the UV emission lines. The SXR signal measured by the SXR increases during reconnection, and also shows a modest change in its spectral energy distribution. However, our steady-state non-LTE models (which assume statistical equilibrium in the excitation/ionization state of the plasma) cannot reliably reproduce the time evolution of the SXR signals, indicating that additional physics-for example, non-Maxwellian particle distributions-must be included in future modeling.

\section{ACKNOWLEDGMENTS}

The authors gratefully acknowledge the technical assistance of S. Palmer and J. Haldeman at Swarthmore, and J. MacFarlane at Prism Computational Sciences, as well as some preliminary modeling done by V. Swisher at Swarthmore. This work was supported by the DOE under Grant No. ER54604, by the NSF Center for Magnetic SelfOrganization (Award No. PHY-0821899), and by a Eugene M. Lang summer research fellowship from the Provost's Office at Swarthmore College.

${ }^{1}$ M. R. Brown, Phys. Plasmas 6, 1717 (1999).

${ }^{2}$ M. R. Brown, C. D. Cothran, and J. Fung, Phys. Plasmas 13, 056503 (2006).

${ }^{3}$ M. R. Brown, C. D. Cothran, M. Landreman, D. Schlossberg, W. H. Matthaeus, G. Qin, V. S. Lukin, and T. Gray, Phys. Plasmas 9, 2077 (2002).

${ }^{4}$ M. R. Brown, C. D. Cothran, M. Landreman, D. Schlossberg, W. H. Matthaeus, G. Qin, V. S. Lukin, and T. Gray, Astrophys. J. Lett. 577, L63 (2002).

${ }^{5}$ C. G. R. Geddes, T. W. Kornack, and M. R. Brown, Phys. Plasmas 5, 1027 (1998).

${ }^{6}$ C. D. Cothran, A. Falk, A. Fefferman, M. Landreman, and M. R. Brown, Phys. Plasmas 10, 1748 (2003).

${ }^{7}$ M. R. Brown, C. D. Cothran, J. Fung, M. Chang, J. Horwitz, M. J. Schaffer, J. Leuer, and E. V. Belova, Phys. Plasmas 13, 102503 (2006).

${ }^{8}$ W. H. Matthaeus, C. D. Cothran, M. Landreman, and M. R. Brown, Geophys. Res. Lett. 32, L23104, DOI:10.1029/2005GL023973 (2005).

${ }^{9}$ Z. Cui, P. Sun, J. Dong, Y. Huang, Q. Wang, B. Fu, J. Lu, W. Chen, X. Duan, X. Ding, Q. Yang, and S. Morita, Plasma Sources Sci. Technol. 10, 298 (2008).

${ }^{10}$ G. Bertschinger, W. Biel, TEXTOR-94 Team, O. Herzog, J. Weinheimer, H.-J. Kunze, and M. Bitter, Phys. Scr. T83, 132 (1999).

${ }^{11}$ N. E. Lanier, S. P. Gerhardt, and D. J. Den Hartog, Rev. Sci. Instrum. 72, 1188 (2001).

${ }^{12}$ K. Widmann, G. V. Brown, M. J. May, M. B. Schneider, H. Chung, D. Hinkel, D. B. Thorn, and P. Beiersdorfer, IEEE Conference Record-IEEE International Conference on Plasma Science, 2005 (IEEE Nuclear and Plasma Sciences Society, Monterey, CA, 2005), Vol. 1, p. 181.

${ }^{13}$ R. Barnsley, I. Coffey, R. Lucock, and M. F. Stamp, Rev. Sci. Instrum. 74, 1969 (2003)

${ }^{14}$ B. C. Stratton, M. Bitter, K. W. Hill, D. L. Hollis, and J. T. Hogan, Fusion Sci. Technol. 53, 431 (2008).

${ }^{15}$ R. Katai, S. Morita, and M. Goto, Rev. Sci. Instrum. 77, 10F307 (2006).

${ }^{16}$ U. Shumlak, R. P. Golingo, B. A. Nelson, and D. J. D. Hartog, Phys. Rev. Lett. 87, 205005 (2001).

${ }^{17}$ K. Matama, M. Yoshikawa, T. Kobayashi, Y. Kubota, and T. Cho, Rev. Sci. Instrum. 77, 10F103 (2006).

${ }^{18}$ M. Mattioli, K. B. Fournier, M. E. Puiatti, M. Valisa, L. Carraro, I. Coffey, 
M. O'Mullane, F. Sattin, and P. Scarin, Plasma Phys. Controlled Fusion 44, 33 (2002).

${ }^{19}$ M. Asif and A. Ikram, Plasma Sources Sci. Technol. 6, 2199 (2004).

${ }^{20}$ M. C. Weisskopf, B. Brinkman, C. Canizares, G. Garmire, S. Murray, and L. P. V. Speybroeck, Publ. Astron. Soc. Pac. 114, 1 (2002).

${ }^{21}$ F. Jansen, D. Lumb, B. Altieri, J. Clavel, M. Ehle, C. Erd, C. Gabriel, M. Guainazzi, P. Gondoin, R. Much, R. Munoz, M. Santos, N. Schartel, D. Texier, and G. Vacanti, Astron. Astrophys. 365, L1 (2001).

${ }^{22}$ C. R. Canizares, D. P. Huenemoerder, D. S. Davis, D. Dewey, K. A. Flanagan, J. Houck, T. H. Markert, H. L. Marshall, M. L. Schattenburg, N. S. Schulz, M. Wise, J. J. Drake, and N. S. Brinkhouse, Astrophys. J. Lett. 539, L41 (2000)

${ }^{23}$ A. C. Brinkman, C. J. T. Gunsing, J. S. Kaastra, R. L. J. van der Meer, R. Mewe, F. Paerels, A. J. J. Raassen, J. J. van Rooijen, H. Bräuninger, W. Burkert, V. Burwitz, G. Hartner, P. Predehl, J.-U. Ness, J. H. M. M. Schmitt, J. J. Drake, O. Johnson, M. Juda, V. Kashyap, S. S. Murray, D. Pease, P. Ratzlaff, and B. J. Wargelin, Astrophys. J. Lett. 530, L111 (2000).

${ }^{24}$ J. W. den Herder, A. C. Brinkman, S. M. Kahn, G. Branduardi-Raymont, K. Thomsen, H. Aarts, M. Audard, J. V. Bixler, A. J. den Boggende, J. Cottam, T. Decker, L. Dubbeldam, C. Erd, H. Goulooze, M. Güdel, P. Guttridge, C. J. Hailey, K. Al Janabi, J. S. Kaastra, P. A. J. de Korte, B. J. van Leeuwan, C. Mauche, A. J. McCalden, R. Mewe, A. Naber, F. B. Paerels, J. R. Peterson, A. P. Rasmussen, K. Rees, I. Sakelliou, M. Sako, J. Spodek, M. Stern, T. Tamura, J. Tandy, C. P. de Vries, S. Welch, and A. Zehnder, Astron. Astrophys. 365, L7 (2001).

${ }^{25}$ M. Gudel and A. Telleschi, Astron. Astrophys. 474, L25 (2007).

${ }^{26}$ N. A. Miller, J. P. Cassinelli, W. L. Waldron, J. J. MacFarlane, and D. H. Cohen, Astrophys. J. 577, 951 (2002).

${ }^{27}$ E. H. Avrett and R. Loeser, Astrophys. J., Suppl. 175, 229 (2008).

${ }^{28}$ D. P. Huenemoerder, Phys. Plasmas 11, 2823 (2004).

${ }^{29}$ A. Telleschi, M. Gudel, K. Briggs, M. Audard, J.-U. Ness, and S. L. Skinner, Astrophys. J. 622, 653 (2005)
${ }^{30}$ P. S. Wojdowski and N. S. Schulz, Astrophys. J. 627, 953 (2005).

${ }^{31}$ M. Landreman, C. D. Cothran, M. R. Brown, M. Kostora, and J. T. Slough, Rev. Sci. Instrum. 74, 2361 (2003).

${ }^{32}$ C. D. Cothran, J. Fung, M. R. Brown, and M. J. Schaffer, Rev. Sci. Instrum. 77, 063504 (2006).

${ }^{33}$ Prism Computational Sciences, Inc., PRISMSPECT, Spectral Analysis Code, 455 Science Drive, Suite 140, Madison, WI, 53711 (www.prism-cs.com/ Software/PrismSpect/PrismSPECT.htm).

${ }^{34}$ J. J. MacFarlane, I. E. Golovkin, P. R. Woodruff, D. R. Welch, B. V. Oliver, T. A. Mehlhorn, and R. B. Campbell, in Proceedings of the Third International Conference on Inertial Fusion Science and Applications, edited by B. A. Hammel, D. D. Meyerhofer, J. M. ter Vehn, and H. Azechi (American Nuclear Society, Monterey, 2004).

${ }^{35}$ H. Suno and T. Kato, At. Data Nucl. Data Tables 92, 407 (2006).

${ }^{36}$ I. E. Golovkin, J. J. MacFarlane, P. Woodruff, J. E. Bailey, G. Rochau, K. Peterson, T. A. Mehlhorn, and R. C. Mancini, J. Quant. Spectrosc. Radiat. Transf. 99, 199 (2006).

${ }^{37}$ Prism Computational Sciences, Inc., Prism atomic physics data, Prism Atomic Data, 455 Science Drive, Suite 140, Madison, WI, 53711 (www.prism-cs.com/Software/AtomicData/AtomicData.htm).

${ }^{38}$ D. E. Osterbrock, Astrophysics of Gaseous Nebulae and Active Galactic Nuclei (University Science, Mill Valley, 1989).

${ }^{39}$ C. D. Cothran, M. Landreman, and M. R. Brown, Geophys. Res. Lett. 30, 1213, DOI:10.1029/2002GL016497 (2003).

${ }^{40}$ J. F. Hansen, Ph.D. thesis, Caltech, 2001.

${ }^{41}$ S. Hokin, R. Fonck, and P. Martin, Rev. Sci. Instrum. 63, 5038 (1992).

${ }^{42}$ V. A. Soukhanovskii, D. Stutman, M. Iovea, M. Finkenthal, H. W. Moos, T. Munsat, B. Jones, D. Hoffman, R. Kaita, and R. Majeski, Rev. Sci. Instrum. 72, 737 (2001).

${ }^{43}$ B. L. Henke, E. M. Gullikson, and J. C. Davis, At. Data Nucl. Data Tables 54, 181 (1993).

${ }^{44}$ J. H. Hubbell, W. J. Veigele, E. A. Briggs, R. T. Brown, D. T. Cromer, and R. J. Howerton, J. Phys. Chem. Ref. Data 4, 471 (1975); 6, 615(e) (1977). 